\title{
A waveguide metasurface based quasi-far-field transverse-electric superlens
}

\author{
Yechuan Zhu ${ }^{1,2,3 \dagger}$, Xiaolin Chen ${ }^{4,5 \dagger}$, Weizheng Yuan ${ }^{1,2}$, Zhiqin $\mathrm{Chu}^{6}$, \\ Kwok-yin Wong ${ }^{5}$, Dangyuan Lei $^{7 *}$ and Yiting $\mathrm{Yu}^{1,2 *}$
}

\begin{abstract}
The imaging capability of conventional lenses is mainly limited by the diffraction of light, and the so-called superlens has been developed allowing the recovery of evanescent waves in the focal plane. However, the remarkable focusing behavior of the superlens is greatly confined in the near-field regime due to the exponential decay of evanescent waves. To tackle this issue, we design a waveguide metasurface-based superlens with an extraordinary quasi-far-field focusing capability beyond the diffraction limit in the present work. Specifically, we analyze the underlying physical mechanism and provide experimental verification of the proposed superlens. The metasurface superlens is formed by an array of gradient nanoslits perforated in a gold slab, and supports transverse-electric (TE) waveguide modes under linearly polarized illumination along the long axis of the slits. Numerical results illustrate that exciting such TE waveguide modes can modulate not only optical phase but also evanescent waves. Consequently, some high-spatial-frequency waves can contribute to the focusing of the superlens, leading to the quasi-far-field super-resolution focusing of light. Under $405 \mathrm{~nm}$ illumination and oil immersion, the fabricated superlens shows a focus spot of $98 \mathrm{~nm}$ (i.e. $1 / 4.13$ ) at a focal distance of 1.49 $\mu \mathrm{m}$ (i.e. 3.68 $\lambda$ ) using an oil immersion objective, breaking the diffraction limit of $\lambda / 2.38$ in the quasi-far field regime. The developed metasurface optical superlens with such extraordinary capabilities promises exciting avenues to nanolithography and ultra-small optoelectronic devices.
\end{abstract}

Keywords: superlens; metasurface; waveguide; quasi-far-field super-resolution focusing; breaking the diffraction limit

Zhu YC, Chen XL, Yuan WZ, Chu ZQ, Wong KY et al. A waveguide metasurface based quasi-far-field transverse-electric superlens. Opto-Electron Adv 4, 210013 (2021).

Concentrating light into a tiny spot has gained increasingly interests in the field of high-resolution optical imaging. However, the imaging capability of a conventional refractive lens is generally restricted to $0.61 \lambda / N A$ ( $\lambda$ being the wavelength of light, $N A$ being the numerical aperture of the lens) due to the diffraction of light waves $^{1}$. In the last two decades, many theoretical and experimental efforts have been made to overcome such limit $^{2}$. In 2000, a perfect lens formed by a slab of a negative refractive index material was conceptually proposed

1Shaanxi Province Key Laboratory of Micro and Nano Electro-Mechanical Systems, Northwestern Polytechnical University, Xi'an 710072, China; ${ }^{2}$ Key Laboratory of Micro/Nano Systems for Aerospace (Ministry of Education), Northwestern Polytechnical University, Xi'an 710072, China; ${ }^{3}$ Shaanxi Province Key Laboratory of Thin Films Technology and Optical Test, Xi'an Technological University, Xi'an 710021, China; ${ }^{4}$ Department of Optical Engineering, School of Electronic Science \& Applied Physics, Hefei University of Technology, Hefei 230601, China; ${ }^{5}$ Department of Applied Biology and Chemical Technology, The Hong Kong Polytechnic University, Hung Hom, Hong Kong 999077, China; 6 Department of Electrical and Electronic Engineering, Joint Appointment with School of Biomedical Sciences, The University of Hong Kong, Hong Kong 999077, China; ${ }^{7}$ Department of Materials Science and Engineering, City University of Hong Kong, Hong Kong 999077, China.

†These authors contributed equally to this work.

*Correspondence: DY Lei, E-mail: dangylei@cityu.edu.hk; YT Yu, E-mail: yyt@nwpu.edu.cn

Received: 2 February 2021; Accepted: 22 March 2021; Published online: 30 September 2021

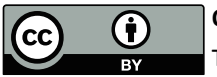

Open Access This article is licensed under a Creative Commons Attribution 4.0 International License.

C The Author(s) 2021. Published by Institute of Optics and Electronics, Chinese Academy of Sciences. 
to focus all Fourier components stemming from strongly enhanced evanescent waves across the lens ${ }^{3}$. Inspired by the concept of the perfect lens, a superlens was developed with an ultrathin silver slab that showed an imaging resolution of $\lambda / 6$ via the excitation of surface plasmons ${ }^{4}$. Moreover, taking the advantage of surface plasmons, super-resolution focusing of light was also realized with various metallic nanostructures ${ }^{5,6}$. However, the working distance of these devices was only tens of nanometers away from itself i.e. in the near-field region, which greatly limited its practical applications. In order to focus light to a greater distance, numerous planar lenses have been proposed based on various nanostructures, such as metallic cross-shaped apertures ${ }^{7}$, metallic slits $^{8-12}$, metallic antennas ${ }^{13,14}$, and dielectric pillars ${ }^{15-21}$. By exploiting those nanostructures to manipulate the phase and amplitude of the incident light, the planar lenses can focus light beyond the near-field regime, holding great promise for practical applications. Nevertheless, none of these previous reports experimentally demonstrates super-resolution focusing in the far field.

Recently, we have theoretically designed a superlens formed by an array of metallic nanoslits and demonstrated quasi-far-field super-resolution focusing ${ }^{22,23}$. In the present work, we apply coupled-mode theory to deeply understand the physical mechanism responsible for the unusual focusing capacity of our superlens and give an experimental demonstration in the quasi-far field region. Figure 1(a) sketches the structure of our waveguide metasurface-based superlens, which comprises of an array of nanoslits with varied widths and constant lengths perforated in a gold slab. The nanoslits are arranged symmetrically with respect to the plane $y=0$, and the width of each slit is elaborately designed based on the theory of wavefront reconstruction and phase modulation. Figure 1(b) illustrates the schematic focusing of the superlens.

When a TE-polarized light beam (with polarization parallel to the nanoslits along the $x$ direction) is normally incident upon the superlens from the glass substrate, it can couple with the waveguide modes of the nanoslits, propagate and radiate into the working dielectric domain. Note that each nanoslit can be assumed to be infinite in the $x$ direction when it is longer than 15 $\mu \mathrm{m}^{24}$. Thus, the nanoslits in the metal slab can be simplified by considering them as parallel-plate metallic waveguides, and the dispersion relation for $\mathrm{TE}_{m}(m=0,1,2$, ...) guided modes is given by:

$$
\frac{w}{2} \sqrt{\varepsilon_{\mathrm{d}} k^{2}-\beta^{2}}=\frac{m \pi}{2}+\arctan \frac{\sqrt{\beta^{2}-\varepsilon_{\mathrm{m}} k^{2}}}{\sqrt{\varepsilon_{\mathrm{d}} k^{2}-\beta^{2}}},
$$

where $w$ is the nanoslit width. $\varepsilon_{\mathrm{m}}$ and $\varepsilon_{\mathrm{d}}$ are the relative permittivities of the metal and the dielectric material in the nanoslit, respectively. $k$ is the free-space wavevector. For each TE mode, the propagation constant $\beta$ can be obtained from Eq. (1). Considering the backward and forward reflections between the input and output interfaces, the electromagnetic field distribution in the $i$ th nanoslit can be expressed in terms of expansion coefficients $\left\{A_{i}, B_{i}\right\}$ as:

$$
E_{i x}(y, z)=\left[A_{i} \mathrm{e}^{\mathrm{j} \beta_{i} z}+B_{i} \mathrm{e}^{-\mathrm{j} \beta_{i} z}\right] f_{i}(y),
$$

where $\beta_{i}$ is the propagation constant, and $f_{i}(y)$ is the eigenmode of the waveguide. By applying the plane-wave expansions (including propagating and evanescent waves) at both sides of the superlens and also matching the boundary conditions for the waveguide modes at a

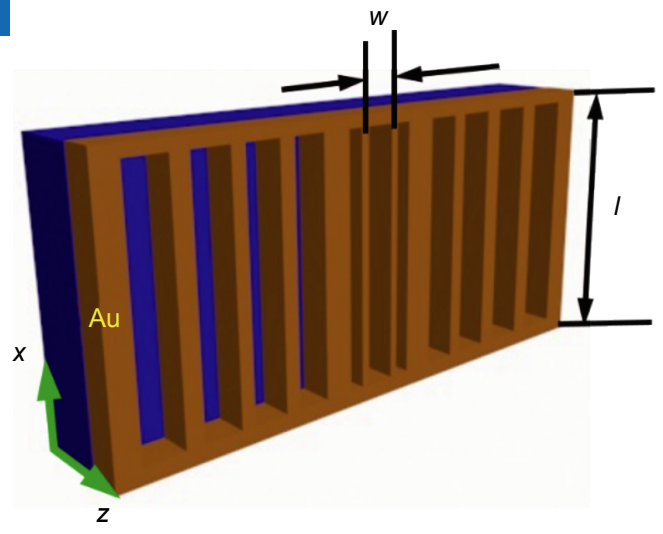

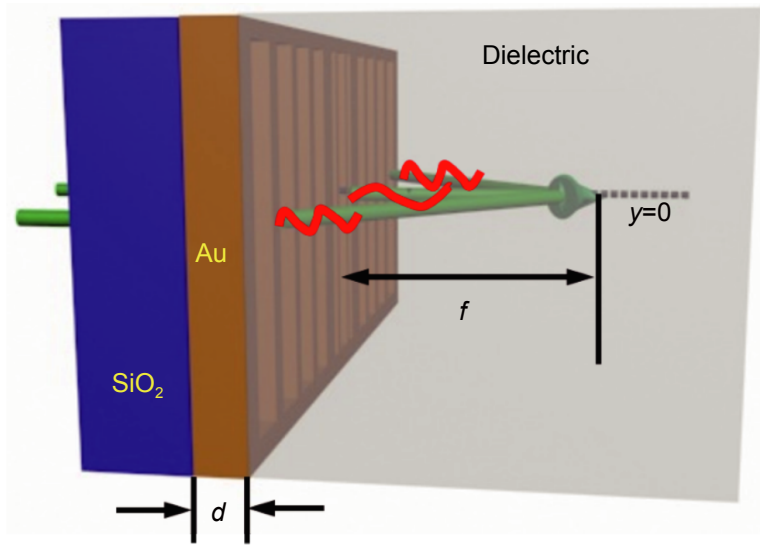

Fig. 1 (a) The illustration of the superlens formed by an array of width-varied nanoslits perforated in a gold film on a glass substrate. All the nanoslits have the same length $l$. The width of each nanoslit $w$ is variable. (b) Schematic focusing of the superlens based on the principle of optical interference under the normal illumination of a TE-polarized plane wave. $d$ is the thickness of gold film and $f$ is the focal distance. 
both the entrance and exit surfaces, we can find a set of linear equations and expansion coefficients that can be analytically calculated ${ }^{25,26}$. In this work, the zeroth mode $\mathrm{TE}_{0}$ is selected for the superlens design to realize the required subwavelength focusing. Within the perfect conductor approximation (i.e. ignoring the skin depth), the electric field and its Fourier spectrum for the $\mathrm{TE}_{0}$ mode $\left(f_{i}(y)=\cos \left(\sqrt{k^{2} \varepsilon_{\mathrm{d}}-\beta_{i}^{2}} y\right)\right)$ in the working dielectric domain can be expressed as following:

$$
\begin{aligned}
& E_{x}\left(k_{y}, z\right)=\frac{1}{2 \pi} \mathrm{e}^{\mathrm{j} \sqrt{k^{2} \varepsilon_{\mathrm{d}}-k_{y}^{2}}(z-d)} \sum_{i=-N}^{N} E_{i} W_{i}\left(k_{y}\right), \\
& E_{x}(y, z)=\frac{1}{2 \pi} \sum_{i=-N}^{N} E_{i} \int_{-\infty}^{+\infty} \mathrm{e}^{\mathrm{j} \sqrt{k^{2} \varepsilon_{\mathrm{d}}-k_{y}^{2}}(z-d)} W_{i}\left(k_{y}\right) \mathrm{e}^{\mathrm{j} k_{y} y} \mathrm{~d} k_{y}
\end{aligned}
$$

where $E_{i}=A_{i} \mathrm{e}^{\mathrm{j} \beta_{i} d}+B_{i} \mathrm{e}^{-\mathrm{j} \beta_{i} d}$ is the modal amplitude of the electric field in the $i$ th nanoslit at the output interface, $d$ is the thickness of the gold film, $k_{y}$ is the wavevector in $y$ direction, and $2 N+1$ is the total number of nanoslits in the superlens. $W_{i}\left(k_{y}\right)$ is the diffraction efficiency of a single nanoslit and can be calculated by

$$
W_{i}\left(k_{y}\right)=\mathrm{e}^{-\mathrm{j} k_{y} a_{i}} \int_{-w_{i} / 2}^{+w_{i} / 2} \mathrm{e}^{-\mathrm{j} k_{y} y} f_{i}(y) \mathrm{d} y,
$$

where $a_{i}$ and $w_{i}$ are the central position and width of the $i$ th nanoslit, respectively. It can be seen from Eq. (3) that the total electric field in the working dielectric domain is the sum of fields produced by arrayed nanoslits and weighted by the modal amplitudes. By tailoring the device parameters, such as the number of nanoslits, and their width and spacing, we can design a targeted field profile.

Now let us design the superlens to achieve subwavelength focusing. According to the theory of wavefront reconstruction and the principle of equal optical path, the focus at a desired distance $f$ from the superlens can be realized, provided that the phase delay of each nanoslit matches the required phase difference $(\Delta \varphi(y)-$ $\Delta \varphi(0))$ as a function of the distance $y$ from the center of the lens, given by

$$
\Delta \varphi(y)-\Delta \varphi(0)=2 p \pi+\frac{2 \pi n_{\mathrm{d}} f}{\lambda}-\frac{2 \pi n_{\mathrm{d}} \sqrt{f^{2}+y^{2}}}{\lambda},
$$

where $\Delta \varphi(y)$ is the phase delay of the nanoslit located at the position $y, \lambda$ is the incident wavelength, $p$ is an arbitrary integer, and $n_{\mathrm{d}}$ is the refractive index of the working dielectric for the superlens. Therefore, the phase delay of each nanoslit is the key to the superlens design.

As pointed out in the previous sections, the phase delay of a nanoslit can be predicted by $\operatorname{Re}\left(\beta_{0}\right) d$, where $\beta_{0}$ is the propagation constant for the $\mathrm{TE}_{0}$ mode and can be calculated by the following equation deduced from Eq. (1) by setting $m=0$ :

$$
\frac{w}{2} \sqrt{\varepsilon_{\mathrm{d}} k^{2}-\beta_{0}^{2}}=\arctan \frac{\sqrt{\beta_{0}^{2}-\varepsilon_{\mathrm{m}} k^{2}}}{\sqrt{\varepsilon_{\mathrm{d}} k^{2}-\beta_{0}^{2}}} .
$$

It can be seen from Eq. (6) that for a specific film thickness $d$ and operating wavelength, the phase delay of a nanoslit can be modulated by the nanoslit width and the working dielectric. On the other hand, by setting the propagation constant to zero, we can find out the cut-off slit width for a TE mode at a given wavelength, below which the mode cannot propagate but decays exponentially with the distance. In one word, the width of a nanoslit should be above the cut-off width for $\mathrm{TE}_{0}$ mode but below that for the $\mathrm{TE}_{1}$ mode to ensure the existence of only $\mathrm{TE}_{0}$ mode in each nanoslit. As a result, the nanoslit width $w$ should meet the following condition:

$$
\frac{2 \arctan \sqrt{-\varepsilon_{\mathrm{m}} / \varepsilon_{\mathrm{d}}}}{k \sqrt{\varepsilon_{\mathrm{d}}}}<w<\frac{\pi+2 \arctan \sqrt{-\varepsilon_{\mathrm{m}} / \varepsilon_{\mathrm{d}}}}{k \sqrt{\varepsilon_{\mathrm{d}}}} .
$$

Based on Eqs. (5) and (7), a superlens immersed in cedar oil with a desired focal length of $f=1.5 \mu \mathrm{m}$ can be designed with a $200 \mathrm{~nm}$ thick gold film at the wavelength of $405 \mathrm{~nm}$. At this wavelength, the permittivity values of gold and cedar oil are $-1.6745+5.7286 \mathrm{i}^{27}$ and $2.2952\left(n_{\mathrm{d}}=\right.$ $1.5150)$, respectively. For a half part of the metasurface superlens beginning from $y=0$, the central positions of the nanoslits are at: $0,0.304,0.910,1.376,1.746,2.086$, 2.408, 2.718, 3.02, 3.316, 3.606, 3.894, 4.180, 4.462, 4.744, and $5.024 \mu \mathrm{m}$. The widths of the first three nanoslits are 190,130 , and $250 \mathrm{~nm}$, respectively, and all the others are $190 \mathrm{~nm}$. All the nanoslits are $15 \mu \mathrm{m}$ long in the $x$ direction. Accordingly, the numerical aperture of the superlens $N A$ is 1.45 .

To understand the focusing property of the designed superlens, full-wave electromagnetic field simulations were performed using the finite-difference time-domain (FDTD) method. Perfectly matched layers as absorbing boundary conditions were employed around the computational domain. A TE-polarized plane wave was defined by setting the amplitude of the electric field component $E_{x}$ to be unit. The size for all the grids was uniformly set to be $2 \mathrm{~nm}$ both in the transverse $y$ direction and in the longitudinal $z$ direction. The simulated electric field intensity distribution of the superlens is illustrated in Fig. 2(a), which verifies the focusing behavior of the designed superlens by producing a bright subwavelength spot. The simulated focal length of the superlens is 1.484 
$\mu \mathrm{m}$, which is close to the designed value $(1.5 \mu \mathrm{m})$. Furthermore, according to the intensity distribution at the focal plane of the superlens as illustrated in Fig. 2(b), the full-width at half-maximum (FWHM) of the focus is 98 $\mathrm{nm}$, namely, $\lambda / 4.13$, well beyond the diffraction limit of $170 \mathrm{~nm}$ (calculated by $0.61 \lambda / N A$, namely, $\lambda / 2.38$ ). Therefore, a super-resolution focus with a focal length of $3.66 \lambda$ is realized by the superlens. Moreover, the simulated transmission efficiency of the superlens is about $40.2 \%$ and the maximum intensity in the focal plane of the focus is 4.03 times larger than the incident intensity. In addition, we also calculated the electric field distribution profiles both in the working dielectric domain (Fig. 2(d)) and at the focal plane (Fig. 2(e)) using the analytical solutions given by Eq. (3). It can be seen that the results agree well with the FDTD simulation results. For simplicity, here the modal amplitude on the exit surface of each nanoslit is obtained by interpolating with the FDTD simulated electric field.

To explore the underlying mechanism of the proposed superlens working in the quasi-far field, we did a special Fourier transformation for the FDTD simulated electromagnetic waves along the $y$ direction. The corresponding Fourier spectrum of electric field as a function of spatial frequency $k_{y}$ (the wavevector in $y$ direction) and position $z$ are given in Fig. 2(c). The blue and green dashed lines mark the positions of the normalized cut-off frequency of conventional lenses $N A / n_{\mathrm{d}}(\approx 0.96)$ and working dielectric $k_{0}\left(k_{0}=2 \pi n_{\mathrm{d}} / \lambda\right)$, respectively. From Fig. 2(c), we can see that some considerable high-spatialfrequency components with $k_{y}>k_{0}$, i.e. beyond the cutoff spatial frequency of working dielectric, locate at the focal spot. And more components with $k_{y}>k_{0} N A / n_{\mathrm{d}}$ are generated at the exit plane of the superlens (at $z=0 \mu \mathrm{m}$ ). These results indicate there are some high-spatial-frequency waves generated and amplified by the superlens. Therefore, the superlens not only provides the components with $k_{y}<k_{0} N A / n_{\mathrm{d}}$, but also brings the high-spatialfrequency components with $k_{y}>k_{0} N A / n_{\mathrm{d}}$, which are normally lost in the foci of traditional refractive lenses, thus leading to the super-resolution focusing of the superlens beyond the diffraction limit. Moreover, the theoretically calculated angular spectrum of electric field is given in Fig. 2(f), which agrees very well with the a
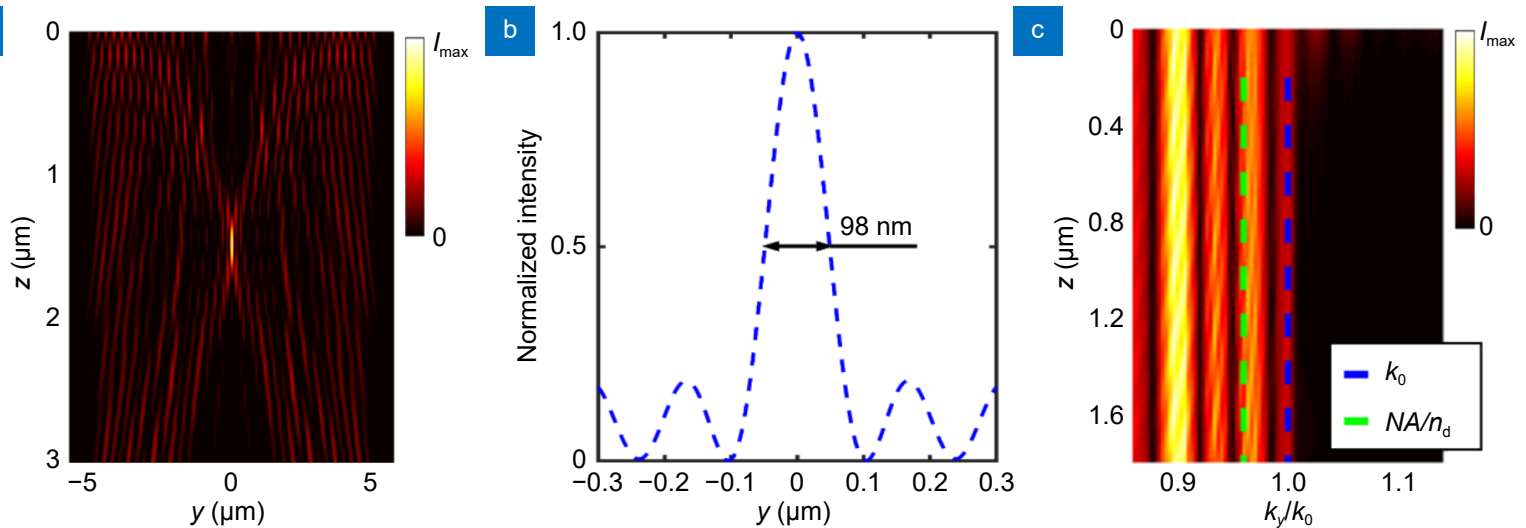

d
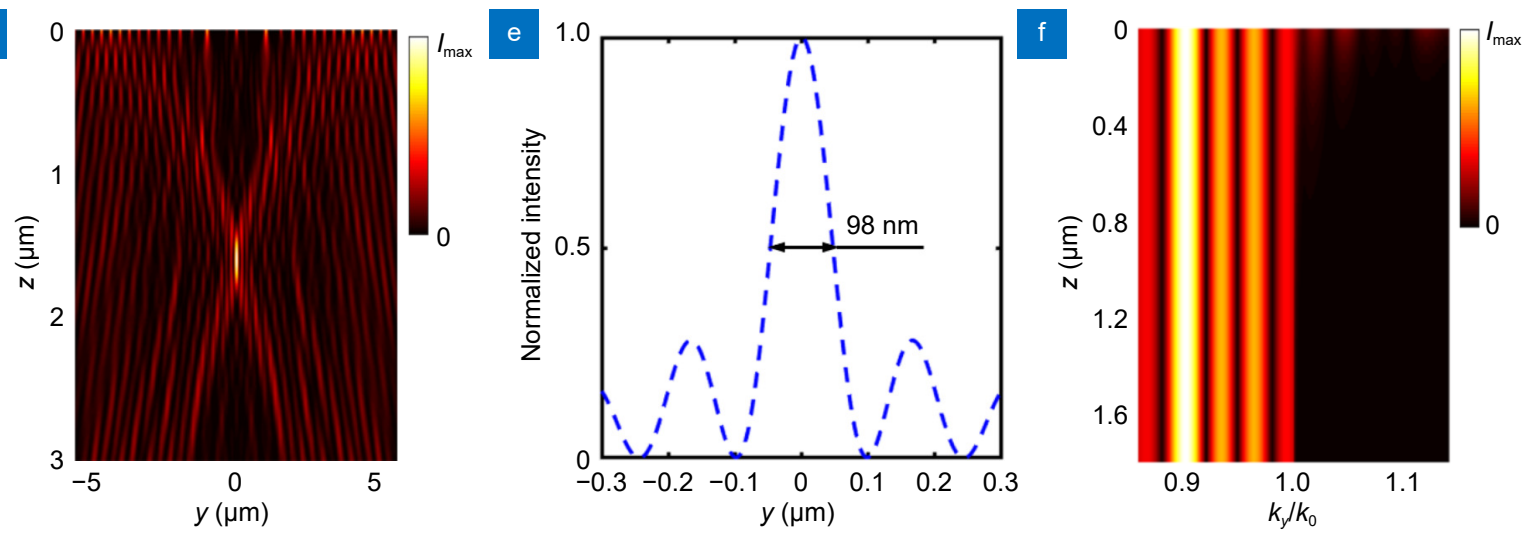

Fig. 2 | Electric field distributions and Fourier spectra of a metasurface superlense. The FDTD simulated intensity distributions (a) in the $y$ - $z$ plane at $x=0,(\mathbf{b})$ at the focal plane, and (c) in $k$-space near the superlens focus. The plane $z=0$ is the exit surface of the superlens. Corresponding theoretically calculated electric field intensity distributions (d) in the $y$-z plane at $x=0,(\mathbf{e})$ at the focal plane, and (f) in $k$-space near the superlens focus. 
numerical results.

After having explored the underlying physics, we performed the experimental work to demonstrate the proposed superlens. Specifically, the superlens was fabricated with the following processes. First, the glass substrate was cleaned using acetone in an ultrasonic bath for 10 minutes. After cleaning, the substrate was washed with the de-ionized water and then dried. Then, a $200 \mathrm{~nm}$ thick gold film as a function layer was deposited on the glass substrate using electron beam evaporation (pressure $<4 \times 10^{-4} \mathrm{~Pa}$; deposition rate: $\left.2 \AA / \mathrm{s}\left(1 \AA=10^{-10} \mathrm{~m}\right)\right)$. Finally, the gold film was nanopatterned by milling through the film using focused ion beam (FIB) (acceleration voltage: $30 \mathrm{kV}$; Ga+ ion beam current: $80 \mathrm{pA}$ ). The resulting structure of the superlens was imaged by scanning electron microscope (SEM), as shown in Fig. 3(a).

We characterized the focusing performance of the fabricated superlens using a Nikon inverted microscope (Nikon Eclipse Ti-U) with oil immersed objective (Nikon CFI LU Plan APO EPI 100×, $N A=0.9$ ). In our optical experiment, a $405 \mathrm{~nm}$ laser was used as the illumination source and expanded to achieve a uniformly distributed beam. After expansion, the uniform beam was polarized parallel to the nanoslits with a linear polarizer (Thorlabs, LPVISE100-A) to achieve a TE-polarized incidence and then irradiated upon the superlens, and the incident beam could be considered locally as a plane wave since the illumination spot was much larger than the size of the superlens structure. A Nikon inverted microscope was employed to find the exit plane of the superlens and collect the transmitted light. Then, the light emitted from the superlens was recorded using a highresolution CCD camera (Olympus, $2560 \times 1280$, the mesh size in the image processing software is $30 \mathrm{~nm}$ ) driven by a piezo-electrical stage with an axial moving resolution of $10 \mathrm{~nm}$ (Physik Instrument, E-816). After fine tuning 400 steps, the intensity distribution of the "focused" superlens was measured, as shown in Fig. 3(b). It is worth noting that we should ensure the light source to be perpendicular to the device in the experiment, since the error in perpendicularity has a great influence on the focusing behavior of the superlens.

As can be seen from Fig. 3(b), the experimentally measured focal length of the superlens is $1.49 \mu \mathrm{m}$ $(3.68 \lambda)$, which is very close to the designed value of $1.5 \mu \mathrm{m}$. Furthermore, according to the intensity distribution at the focal plane of the superlens as illustrated in Fig. 3(c), the experimentally measured FWHM of the superlens also agrees very well with the simulated result. The FWHMs are all $98 \mathrm{~nm}$, namely $\lambda / 4.13$, which is beyond the diffraction limit of $\lambda / 2.38$. Therefore, quasi-farfield super-resolution focusing based on the TE superlens is experimentally verified. It is noted that the superlens we developed demonstrates great advantages over the transverse-magnetic (TM) lens which can also operate in the far-field zone ${ }^{9}$. On the one hand, due to the difference between plasmonic waveguide modes and TE guided modes in generating phase delay, the width of the narrowest nanoslit for our superlens is $130 \mathrm{~nm}$ and the corresponding depth-to-width ratio is less than 2 . In contrast, the narrowest nanoslit width for the TM lens is $80 \mathrm{~nm}$ with the depth-to-width ratio of 5:1. Therefore, our superlens is more favorable for the practical nanofabrication. On the other hand, the TE superlens we developed can realize super-resolution focusing of light at the quasi-far-field zone by invoking high-spatial-frequency components, which cannot be achieved with the TM lens because the high-spatial-frequency surface a

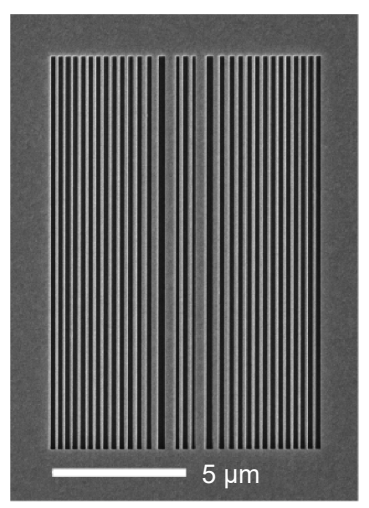

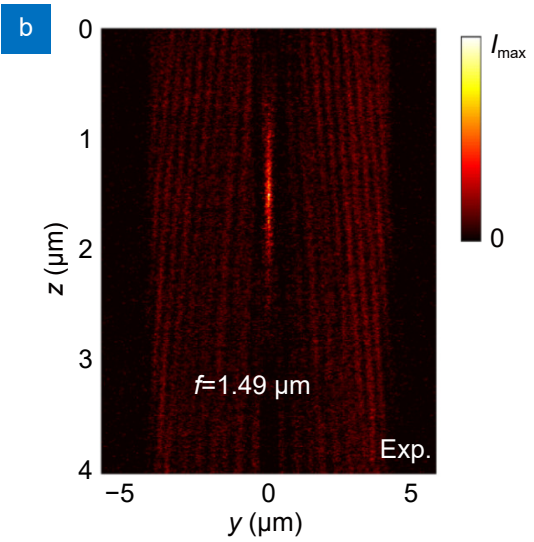

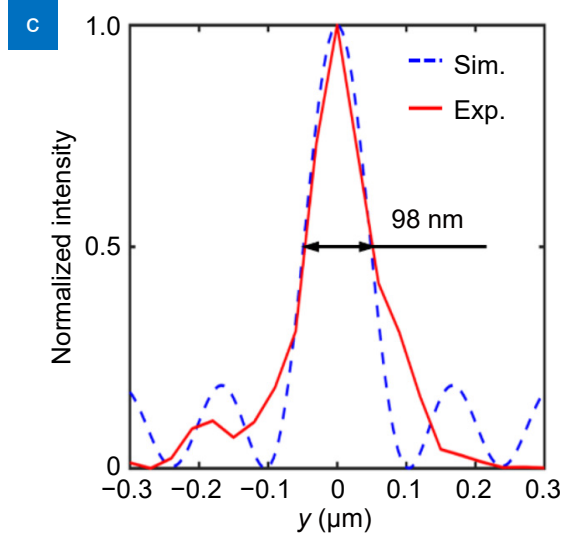

Fig. 3 | Experimental demonstration of the quasi-far-field super-resolution focusing superlens. (a) A scanning electron micrograph of the fabricated superlens using FIB. (b) Measured intensity distributions in the $y-z$ plane at $x=0$. (c) The measured and FDTD simulated intensity distribution at the focal plane. 
waves like SPPs are localized in the near-field zone and decay rapidly from the surface.

In conclusion, we have designed a quasi-far-field super-resolution focusing superlens consisting of nanoslits milled in a gold film via the excitation of TE waveguide modes working at a visible wavelength region. Meanwhile, the experimental measurements on the fabricated superlens show excellent agreements with the numerical simulations, being consistent with our theoretical predictions. More importantly, our superlens can not only provide low-spatial-frequency components, but also bring high-spatial-frequency components that are normally lost in the foci of traditional refractive lenses. The latter is the key to overcome the diffraction limit for the super-resolution focusing using our superlens. Furthermore, the proposed method can be promoted to construct focusing devices beyond the diffraction limit at other wavelengths. Our first experimental demonstration is a crucial step in the realization of this super-resolution focusing technology for many potential applications in optoelectronics and biomedical imaging.

\section{References}

1. Rayleigh SRSL. XV. On the theory of optical images, with special reference to the microscope. London, Edinb, Dublin Philos Mag J Sci 42, 167-195 (1896).

2. Zhang X, Liu ZW. Superlenses to overcome the diffraction limit. Nat Mater 7, 435-441 (2008).

3. Pendry JB. Negative refraction makes a perfect lens. Phys Rev Lett 85, 3966-3969 (2000).

4. Fang $\mathrm{N}$, Lee $\mathrm{H}$, Sun $\mathrm{C}$, Zhang $\mathrm{X}$. Sub-diffraction-limited optical imaging with a silver superlens. Science 308, 534-537 (2005).

5. Pan L, Park $Y$, Xiong $Y$, Ulin-Avila E, Wang $Y$ et al. Maskless plasmonic lithography at $22 \mathrm{~nm}$ resolution. Sci Rep 1, 175 (2011).

6. Gramotnev DK, Bozhevolnyi SI. Nanofocusing of electromagnetic radiation. Nat Photon 8, 13-22 (2014).

7. Ling L, Goh XM, McGuinness LP, Roberts A. Plasmonic lenses formed by two-dimensional nanometric cross-shaped aperture arrays for Fresnel-region focusing. Nano Lett 10, 1936-1940 (2010).

8. Shi HF, Wang CT, Du CL, Luo XG, Dong XC et al. Beam manipulating by metallic nano-slits with variant widths. Opt EXpress 13, 6815-6820 (2005).

9. Verslegers L, Catrysse PB, Yu ZF, White JS, Barnard ES et al. Planar lenses based on nanoscale slit arrays in a metallic film. Nano Lett 9, 235-238 (2009).

10. Yu YT, Zappe H. Effect of lens size on the focusing performance of plasmonic lenses and suggestions for the design. Opt Express 19, 9434-9444 (2011).

11. Yu YT, Zappe H. Theory and implementation of focal shift of plasmonic lenses. Opt Lett 37, 1592-1594 (2012).

12. Zhu YC, Yuan WZ, Yu YT, Diao JS. Metallic planar lens formed by coupled width-variable nanoslits for superfocusing. Opt Express 23, 20124-20131 (2015).
13. Yu NF, Genevet P, Kats MA, Aieta F, Tetienne JP et al. Light propagation with phase discontinuities: generalized laws of reflection and refraction. Science 334, 333-337 (2011).

14. Ni XJ, Emani NK, Kildishev AV, Boltasseva A, Shalaev VM. Broadband light bending with plasmonic nanoantennas. Science 335, 427 (2012).

15. Lin DM, Fan PY, Hasman E, Brongersma ML. Dielectric gradient metasurface optical elements. Science 345, 298-302 (2014).

16. Aieta F, Kats MA, Genevet P, Capasso F. Multiwavelength achromatic metasurfaces by dispersive phase compensation. Science 347, 1342-1345 (2015).

17. Khorasaninejad M, Chen WT, Devlin RC, Oh J, Zhu AY et al. Metalenses at visible wavelengths: diffraction-limited focusing and subwavelength resolution imaging. Science $\mathbf{3 5 2}$, 1190-1194 (2016).

18. Groever B, Chen WT, Capasso F. Meta-lens doublet in the visible region. Nano Lett 17, 4902-4907 (2017).

19. Chen WT, Zhu AY, Sanjeev V, Khorasaninejad M, Shi ZJ et al. A broadband achromatic metalens for focusing and imaging in the visible. Nat Nanotechnol 13, 220-226 (2018).

20. Wang SM, Wu PC, Su VC, Lai YC, Chen MK et al. A broadband achromatic metalens in the visible. Nat Nanotechnol 13, 227-232 (2018).

21. Park JS, Zhang SY, She AL, Chen WT, Lin P et al. All-glass, large metalens at visible wavelength using deep-ultraviolet projection lithography. Nano Lett 19, 8673-8682 (2019).

22. Zhu YC, Yuan WZ, Li WL, Sun H, Qi KL et al. TE-polarized design for metallic slit lenses: a way to deep-subwavelength focusing over a broad wavelength range. Opt Lett 43, 206-209 (2018).

23. Zhu YC, Zhou S, Wang ZH, Yu YT, Yuan WZ et al. Investigation on super-resolution focusing performance of a TE-polarized nanoslit-based two-dimensional lens. Nanomaterials 10, 3 (2020).

24. Pang Y, Genet C, Ebbesen TW. Optical transmission through subwavelength slit apertures in metallic films. Opt Commun 280, 10-15 (2007).

25. Garcia-Vidal FJ, Martin-Moreno L, Ebbesen TW, Kuipers L. Light passing through subwavelength apertures. Rev Mod Phys 82, 729-787 (2010).

26. Takakura $Y$. Optical resonance in a narrow slit in a thick metallic screen. Phys Rev Lett 86, 5601-5603 (2001).

27. Johnson PB, Christy RW. Optical constants of the noble metals. Phys Rev B 6, 4370-4379 (1972).

\section{Acknowledgements}

We acknowledge the financial support by the National Natural Science Foundation of China (52075410, 51975483, 51622509), the Fundamental Research Funds for the Central Universities (31020190504001), the 111 Project (B13044), the Dean Fund (2019GDYJY05), the Collaborative Innovation Center Project of Shaanxi Provincial Department of Education (20JY031), the Natural Science Basic Research Plan in Shaanxi Province of China (2018JQ6012), the Hong Kong Polytechnic University through the "Life Science Research" project (1-ZVH9), and the City University of Hong Kong (9610456). We are also grateful to the useful discussion with Dr. Meng Qiu from the Department of Electrical Engineering at The Hong Kong Polytechnic University.

\section{Competing interests}

The authors declare no competing financial interests. 